\title{
A case of tension pneumocephalus with Mount Fuji sign
}

\author{
Amina Sultana ${ }^{1}$, Mir Atiqur Rahman ${ }^{1}$, Mohammad Omar Faruq², Mohammad Ashraful Haque ${ }^{1}$, Kazi Taimur \\ Rahman $^{1}$, Chowdhury Asif Mahmud ${ }^{1}$
}

A 77 years old man, known hypertensive, diabetic, CKD stage 2, admitted in ICU of a tertiary care hospital in Dhaka through the emergency room (ER) with the complaints of altered level of consciousness, right sided weakness and slurring of speech for three hours. He had no history of headache, vomiting and convulsion. On examination he was unconscious grade 3, pupil bilaterally equal, normal in size and reacting to light, planter reflex: flexor on left \& extensor on right side, CT scan of head on admission showed acute haematoma in left cerebral hemisphere with intra ventricular extension (Figure 1). Craniotomy with total removal of arterio-venous malformation (AVM) with insertion of left sided external ventricular drain (EVD) was done.

On $2^{\text {nd }}$ post operative day (POD) patient developed repeated generalized tonic clonic convulsion (GTCS) which was controlled with diazepam, Phenytoin, and Levetiracetam . CT scan of head was repeated on the same day which showed significant air within the subdural space along the fronto-temporal convexity (Mount Fuji sign Fig 2) \& communication with the left sided frontal sinus (Fig 3). Evidence of left sided craniotomy with small residual haemorrhage with surrounding hypo density in left frontotemporo- parietal lobes with slight compression of left lateral ventricle was also noted.

Patient was on mechanical ventilator. On 7th POD he was successfully weaned from ventilator, he was kept on T- piece for tracheal toileting. Follow- up CT was done which revealed much reduced air content within the subdural space. Subdural collection is seen in the convexity of both fronto- temporoparietal region.

On $12^{\text {th }}$ POD patient was transferred out of ICU in stable condition.

\section{Discussion :}

Pneumocephalus (PNC) refers to the presence of intracranial gas, and in the vast majority of cases the gas is air. PNC can be divided by location, extra-axial, epidural, subdural, subarachnoid; intra-axial, parenchymal, intra-ventricular, intra-vascular etc.

\footnotetext{
1. Intensivist, General ICU, United Hospital Limited.

2. Consultant, General ICU, United Hospital Limited.
}

\section{Corresponding Author:}

Dr. Amina Sultana

MBBS , MD (CCM)

Intensivist, GICU, United Hospital Limited

Plot:15,Rd:71,Gulshan 2, Dhaka, Bangladesh

email:aminasultana95@yahoo.com

Contact :+8801732955192
Tension PNC is a neurosurgical emergency that occurs when subdural air causes a mass-effect over the underlying brain parenchyma, often from a ball-valve mechanism causing one-way entry of air into the subdural space. ${ }^{1}$ Clinical presentation includes headache, nausea and vomiting, seizures, hemiparesis, dizziness, obtundation and depressed neurological status ${ }^{2}$. CT scans of patients with a tension PNC typically show air that compresses the frontal lobes of the brain, which results in a tented appearance of the brain in the skull known as the Mount Fuji sign. ${ }^{3,4,5}$ The name is derived from the resemblance of the brain to Mount Fuji in Japan, a volcano known for its symmetrical cone. In typical cases, there is a symmetrical depression near the midline (such as the crater of a volcano), due to intact bridging veins. ${ }^{5}$ The sign was first described by a team of Japanese neurosurgeons. ${ }^{6}$

Trauma is the most common cause of PNC, accounting for $75 \%$ of cases. Infection, most common chronic otitis media, accounted for $9 \%$ of the cases reviewed. ${ }^{7}$ Common causes of PNC are cranial or spinal surgery as well as some ENT operations, such as paranasal sinus surgery, nasal septum resection, or nasal polypectomy. ${ }^{8}$

PNC usually gets absorbed without any clinical manifestations. The conservative treatment involves placing the patient in the Fowler position of $30^{\circ}$, avoiding Valsalva maneuver (coughing and sneezing), administering pain and antipyretic medications to prevent hyperthermia, and osmotic diuretics.

When clinical signs appear, such as intracranial hypertension or impaired consciousness that endangers the life of the patient, treatment consists of emergent decompression to alleviate pressure on the brain parenchyma.

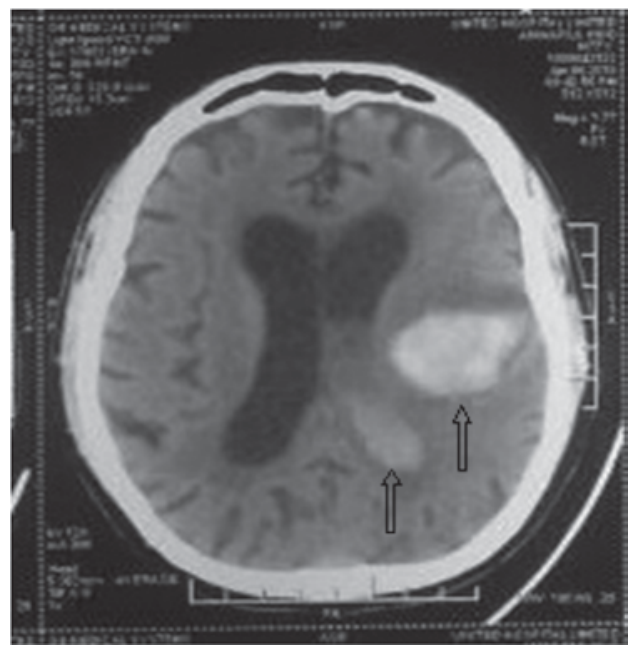

Figure 1: Parenchymal \& intraventricular Haemorrhage (arrows) 


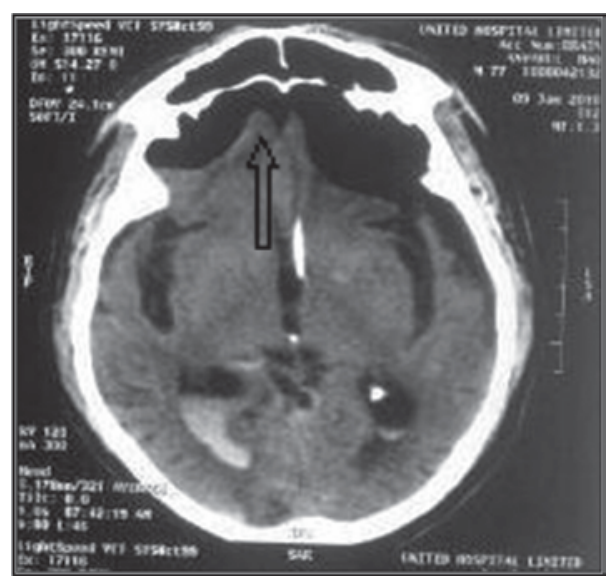

Figure 2 : Mount Fuji sign (arrow)

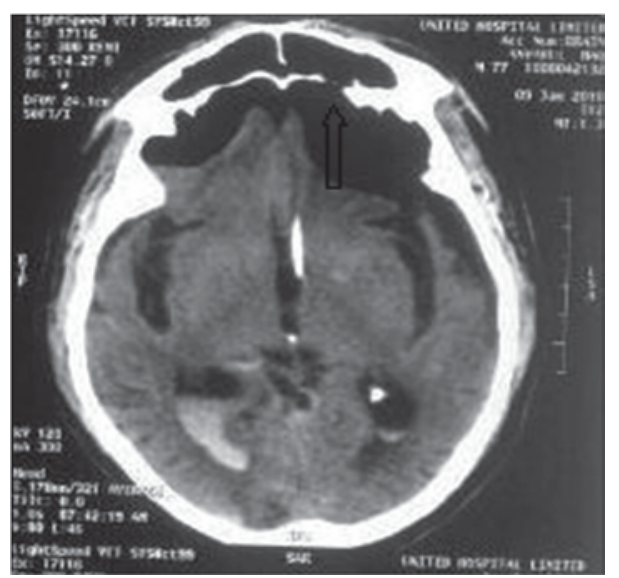

Figure 3: Communication with frontal sinus (arrow)

\section{References:}

1. Dandy WE. Pneumocepahlus (intracranial pneumatocele or aerocele) Arch Surg. 1926;132:949-82.

2. Bernstein AL, Cassidy J, Duchynski R, Eisenberg SS. Atypical headache after prolonged treatment with nasal continuous positive airway pressure. Headache. 2005;45:609-11.

3. Michel SJ . The Mount Fuji sign. Radiology 2004. 232 (2): 449-50.

4. Sadeghian H . Mount Fuji sign in tension pneumocephalus. Archives of Neurology. 2000;57 (9): 1366.

5. Heckmann JG, Ganslandt O. Images in clinical medicine. "The Mount Fuji sign". The New England Journal of Medicine.2004; 350 (18): 1881.

6. Ishiwata Y, Fujino H, Kubokura T, Tsubone K, Sekino T, Fujitsu K . Subdural tension pneumocephalus following surgery of chronic subdural hematoma.No shinkei geka. Neurological surgery (in Japanese) 1987; 15 (4): 419-24.

7. Markham JW. The clinical features of pneumocephalus based upon a survey of 284 cases with report of 11 additional cases. Acta Neurochir (Wien) 1967;16:1-78.

8. Solomiichuk VO, Lebed VO, Drizhdov KI. Posttraumatic delayed subdural tension pneumocephalus. Surg Neurol Int. 2013;4:37. 